\title{
COMPLEXIDADE DE LEMPEL-ZIV NA ANÁLISE DO PARTICIONAMENTO DO POLIETILENOGLICOL NO NANOPORO DE ALFA-HEMOLISINA
}

\author{
Gesilda F. Neves ${ }^{1}$, Dijanah C. Machado ${ }^{2}$, Carlos M. M. Carneiro ${ }^{2}$, Luiz H. A. Consoni ${ }^{2}$, \\ Cláudio G. Rodrigues ${ }^{2}$, Romildo A. Nogueira ${ }^{1 *}$ \\ ${ }^{1}$ Departamento de Morfologia e Fisiologia Animal, UFRPE, ${ }^{2}$ Departamento de Biofísica e Radiobiologia, UFPE \\ *gesildaflorenco@gmail.com
}

\begin{abstract}
INTRODUÇÃO
Métodos estatísticos baseados em dinâmica não linear são amplamente empregados na análise e descrição adequada de processos nas áreas de química, física e biologia. 0 aumento na capacidade de processamento computacional, juntamente com o desenvolvimento de linguagens de programação e algoritmos possibilitaram a disponibilização de programas de simulação e previsão em processos que há alguns anos seriam inexequíveis. 0 mé todo da complexidade de Lempel Ziv (CLZ), permite calcular a complexidade de uma série temporal sem a necessidade de longos segmentos de dados. Uma série temporal corresponde a um conjunto de dados coletados em intervalos regulares de tempo. 0 valor da CLZ correlaciona-se com a estocasticidade ou determinismo de uma série temporal. Séries temporais com o CLZ próximo de 1 são consideradas aleatórias; CLZs próximos a zero representam séries determinísticas (ABOY et al., 2006). Este método foi empregado em vários estudos para análise e inferência da complexidade em: segmentos de DNA (NUNES, 2014), ritmicidade elétrica pancreática in silico (NEVES et al., 2014), déficits cognitivo em pacientes esquizofrê nicos (IBAÑEZ-MOLINA et al., 2017). Séries temporais podem ser obtidas através da aquisição de registros de corrente iônica gerada devido ao fluxo de íons através de nanoporos protéicos (HILLE,2001). A alfa-hemolisina( $\mathrm{aHL}$ ) é o principal nanoporo protéico empregado no biossensoriamento estocástico (REINER et al., 2012). 0 biossensoriamento estocástico é uma abordagem que se baseia na observação de eventos de ligação entre as moléculas individuais de analitos e um único receptor(AGUIAR et al., 2015). Este nanoporo tem sido empregado na detecção de fármacos (KANG et al., 2006), polímeros(RODRIGUES et al., 2008, 2011) e até no sequenciamento de DNA (DING et al., 2010; FENG et al., 2015). O mecanismo de detecção ocorre com a entrada ou translocação do analito através do lúmen aquoso do nanoporo da alfa-hemolisina. Cada molécula entrante ou translocante produz uma variação discretizada (bloqueio) na corrente iônica. O polietilenoglicol (PEG) foi usado como analito. O bloqueio é caracterizado por uma amplitude e um intervalo temporal, que representa o tempo de permanência (TP) do PEG no nanoporo. Outro parâmetro importante no biossensoriamento estocástico é o intervalo temporal que o PEG não está presente no lú men do nanoporo, ou seja, é o tempo de ausência (TA) (FIGURA 1). Neste contexto empregamos o método da CLZ na análise das séries temporais TP ou TA visando inferir se o processo de particionamento do PEG no sistema solução/nanoporo é um processo estocástico ou determinístico.
\end{abstract}

\section{MATERIAIS E MÉTODOS \\ 1.Experimental}

Todas as bicamadas lipídicas planas livres de solvente foram confeccionadas conforme as técnicas convencionais de construção de membranas (MONTAL \& MUELLER, 1972). Esta técnica consiste basicamente na formação de uma bicamada lipídica por aposição de dois filmes monomoleculares de lipídeo sintético, num orifício de uma partição de Teflon $^{\circledast}$ (Politetrafluoretileno) que separa dois compartimentos de uma câmara experimental também de Teflon ${ }^{\circledR}$, contendo soluções aquosas. Foram adicionados em cada hemicâmara aproximadamente $10 \mu \mathrm{l}$ de uma solução de diftanoil glicerofosfocolina $2 \%(\mathrm{p} / \mathrm{v})$ em hexano. Decorridos aproximadamente 10 minutos, com a evaporação do hexano, ocorreu à formação espontânea dos filmes lipídicos monomoleculares na superfície da solução aquosa de cada compartimento. Posteriormente o menisco do líquido de um dos compartimentos foi elevado por adição de mais solução, formando a primeira monocamada. Este mesmo procedimento foi realizado no compartimento oposto para a formaçã o da bicamada. A formação da membrana foi monitorada usando lupa binocular e principalmente pelo aumento da corrente capacitiva basal. Após a construção da bicamada lipídica realizou-se a incorporação de um único nanoporo pela adição da a-hemolisina na solução de uma das hemicamaras. Posteriormente, adicionou-se o polietilenoglicol (PEG 1294) ao compartimento oposto da câmara experimental e registrou-se a corrente iônica fluente através do nanoporo. Todos os experimentos foram realizados em condições de fixação de voltagem $( \pm 100 \mathrm{mV}, \Delta=20 \mathrm{mV})$, solução $(\mathrm{KCl} 4 \mathrm{M}$, Tris 5 $\mathrm{mM}, \mathrm{pH} 7,5$ ) e $23 \pm 2{ }^{\circ} \mathrm{C}$. Um condutivímetro (Radiometer Analytical, CDM230) foi utilizado na determinação da condutividade de todas as soluções utilizadas nos experimentos. Com a finalidade de minimizar a interferência de perturbações mecânicas e eletromagnéticas, toda a montagem do sistema experimental foi mantida sobre uma mesa de amortecimento de alta performance (TMC 63-500, USA) e blindada por uma gaiola de Faraday. Em todos os experimentos o sistema utilizado para estimulação, monitoração e aquisição dos registros era constituído por um gerador de onda triangular, um amplificador de patch clamp Axopatch 200B (Molecular devices, Foster City, CA), uma placa conversora analógico-digital (PCl 6024E da National Instruments Corporation ou DIGIDATA 1440 da Molecular Devices) acoplada a um microcomputador IBM PC compatível. Os registros de corrente iônica através dos nanoporos foram processados a $15 \mathrm{kHz}$ 
por um filtro Bessel (Model 902, Frequency Devices, Haverhill, MA) e aquisitados a 100 ou $250 \mathrm{kHz}$. A conexão das soluções da câmara ao sistema de medidas elétricas se deu através de pontes salinas do tipo Ágar- $\mathrm{KCl}(3 \%$ em peso de Ágar em $\mathrm{KCl} 3 \mathrm{M}$ ) e eletrodos de pratacloreto de prata $(\mathrm{Ag} / \mathrm{AgCl})$. A alfa-toxina foi adquirida da Calbiochem (USA) e o diftanoil glicerofosfocolina da Avanti polar Lipids(USA).

\section{Teórico}

2.1. QUB: Os registros de corrente iônica foram segmentados em sé ries temporais de TP's e TA's através do Software QuB (www.qub.buffalo.edu) e analisado pelo método do CLZ.

2.2. Lempel-Ziv: 0 Lempel-Ziv é um método de cálculo de complexidade e avaliação da aleatoriedade em um sinal unidimensional ou sequências finitas. É considerada uma medida não paramétrica de complexidade que reflete o padrão da série ( LEMPEL; ZIV, 1976; ABÁSOLO et al., 2006; GÓMEZ et al., 2016).Para calcular a CLZ é necessário transformar a série temporal em uma sequência binária que é gerada a partir da comparação de cada ponto de tempo da série com uma média de todos os pontos na série. Se o valor do ponto é maior do que a média, passa a ser representado por 1 , se é menor do que a média será representado por 0 . Deste modo obtém-se uma sequência formada por 1 e 0 (ABÁ SOLO et al., 2006).

A complexidade de uma série pode ser medida da seguinte forma: Seja uma série $X=x(1), x(2), \ldots, x(N)$ que é convertida numa sequê ncia 0-1 gerando um $P=s(1), s(2), \ldots, s(N)$, com $s(i)$ definida por:

$$
s_{(1)}= \begin{cases}0, \text { se } & s_{(i)}<\text { média } \\ 1, \text { se } & s_{(i)} \geq \text { média }\end{cases}
$$

Depois, esta cadeia de caracteres $(P)$ é escaneado da esquerda para a direita e um contador de complexidade $C(n)$ é aumentado em uma unidade cada vez que uma nova subsequência de caracteres consecutivos é encontrada no processo de escaneamento. Finalmente, $c(n)$ é normalizado de modo a obter uma medida de complexidade independente do comprimento da sequência. Para uma conversão binária, o limite superior de $c(n)$ é dado por $b(n)=$ $n / \log _{2}(n)$, e $c(n)$ pode ser normalizado via $b(n)$ :

$$
C L Z=\frac{c(n)}{b(n)}
$$

0 resultado do cálculo da CLZ é entre 0 e 1 . Quanto mais próximo de 1 for o resultado maior a CLZ e mais aleatório será o comportamento do sinal, quanto mais próximo o resultado for de 0 menor será a CLZ, indicando que a série tem maior auto-similaridade ( $A B O Y$ et al., 2006; NEVES et al., 2014;GÓMEZ et al., 2016). Os resultados foram analisados pelo teste estatístico de Kruskal-Wallis e post hoc de Dunn.

\section{RESULTADOS E DISCUSSÃO}

A figura 1 representa o registro típico da corrente iônica através do nanoporo da aHL na presença de PEG1294 na concentração de $2 \mathrm{mM}$. Nota-se que os bloqueios (variações discretizadas) correspondem a presença de uma molécula do PEG no lúmen do nanoporo. Similarmente a outros relatos (ROBERTSON et al., 2007; RODRIGUES et al., 2008) a amplitude dos bloqueios é praticamente constante e se deve a monodispersidade do polímero.

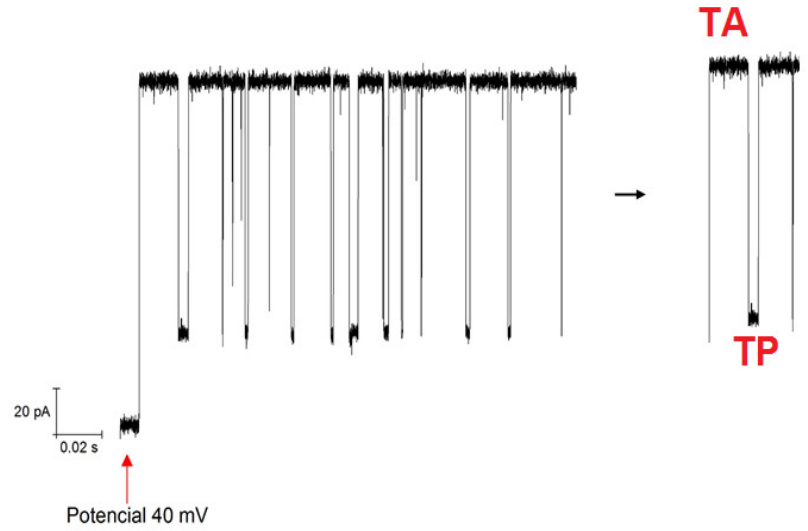

Figura 1. Registro da corrente iônica do nanoporo de aHL na presença de PEG1294. A entrada de uma molécula do PEG provoca o bloqueio temporário da corrente iônica, enquanto que a sua saída resulta no retorno da corrente iônica ao seu maior valor. Em destaque: segmento representativo do tempo de ausência (TA, em azul) e do tempo de permanência (TP, em vermelho) do PEG no interior do nanoporo. Potencial: 40 $\mathrm{mV}$. Concentração do PEG $=2 \mathrm{mM}$.

Pela escassez de trabalhos que infere matematicamente as propriedades estocásticas do bloqueio e desbloqueio, utilizamos a CLZ para ajudar a esclarecer o perfil da translocação em nanoporos. O Lempel-Ziv é uma medida bastante usada para caracterizar a complexidade em sinais biológicos(HU; GAO; PRINCIPE, 2006; HUDETZ et al., 2016). Movileanu et al (2005) observaram o comportamento estocástico de polímeros confinados em nanoporos unitários. O CLZ é um método de cálculo de complexidade e avaliaçã o da aleatoriedade em um sinal unidimensional ou sequências finitas. Os altos valores de CLZ (aproximadamente igual a 1) obtidos para sequência dos tempos de permanência nos estados abertos e bloqueados pode sugerir, matematicamente, que os nanoporos de a $\mathrm{HL}$, nas condições estudadas neste trabalho, se comportam como um biossensor estocástico. As análises com a CLZ mostraram uma alta complexidade nas duas concentrações de PEG testadas e em todas as voltagens aplicadas. Não houve diferenças significativas nos valores de CLZ nas diferentes voltagens e concentrações para um $p<0.05$ (Tabela 1).

Tabela 1. Resultado obtido a partir do algoritmo da CLZ nas concentrações de 1 e $2 \mathrm{mM}$ e

\begin{tabular}{|c|c|c|c|c|}
\hline \multirow{3}{*}{ Voltagem } & \multicolumn{4}{|c|}{ Concentração } \\
\hline & \multicolumn{2}{|c|}{$1 \mathrm{mM}$} & \multicolumn{2}{|c|}{$2 \mathrm{mM}$} \\
\hline & TP & TA & TP & TA \\
\hline $20 m V$ & $0.9662 \pm 0.0051$ & $0.8459 \pm 0.0516$ & $0.9760 \pm 0.0104$ & $0.8965 \pm 0.0174$ \\
\hline $40 m V$ & $0.9704 \pm 0.0007$ & $0.8717 \pm 0.0167$ & $0.9762 \pm 0.0177$ & $0.8937 \pm 0.0091$ \\
\hline $60 m V$ & $0.948 \pm 0.02720$ & $0.9095 \pm 0.0101$ & $0.9669 \pm 0.0088$ & $0.9114 \pm 0.0051$ \\
\hline $80 m V$ & $0.9211 \pm 0.0715$ & $0.9075 \pm 0.0597$ & $0.9656 \pm 0.0012$ & $0.9290 \pm 0.0125$ \\
\hline $100 \mathrm{mV}$ & $0.9500 \pm 0.0143$ & $0.9354 \pm 0.0315$ & $0.9557 \pm 0.0166$ & $0.9546 \pm 0.0114$ \\
\hline
\end{tabular}
voltagens de $20-100 \mathrm{mV}$ (média \pm desvio padrão), $\mathrm{p}<0.05$.

\section{CONCLUSÕES}

O particionamento do polietilenoglicol monodisperso 1294 no sistema solução/nanoporo da $\alpha \mathrm{HL}$ é modelado por um processo estocástico de alta complexidade

\section{REFERÊNCIAS}

ABÁSOLO, D. et al. Analysis of EEG background activity in Alzheimer's disease patients with Lempel-Ziv complexity and central tendency measure. Medical Engineering and Physics, v. 28, n. 4, p. 315-322, 2006. 
ABOY, $M$. et al. Interpretation of the Lempel-Ziv complexity measure in the context of biomedical signal analysis. IEEE Transactions on Biomedical Engineering, v. 53, n. 11, p. 22822288, 2006.

AGUIAR, J. P. et al. Biossensoriamento estocástico via nanoporo proteico individual no desenvolvimento de ferramentas analíticas. Quimica Nova, v. 38, n. 6, p. 817-827, 2015.

NUNES, Ciro Alves Justino. Algoritmo de Lempel-Ziv aplicado à classificação quantitativa de autômatos celulares. 2014. $127 \mathrm{f}$. Dissertacão (Mestrado em Ciências Exatas e da Terra) Universidade Federal de Uberlândia, Uberlândia, 2014.

DING, K.J. et al. Progress of Research on Nanopore-macromolecule Detection. Chinese Journal of Analytical Chemistry, v. 38, n. 2, p. 280-285, 2010.

FENG, Y. et al. Nanopore-based fourth-generation DNA sequencing technology. Genomics, Proteomics and Bioinformatics, v. 13, n. 1, p. 4-16, 2015.

GÓMEZ, C. et al. Characterization of EEG patterns in brain-injured subjects and controls after a Snoezelen ${ }^{\circledR}$ intervention. Computer Methods and Programs in Biomedicine, v. 136, p. 1-9, 2016.

HILLE,B.Ion Channels of excitable Membranes.SINAUER ASSOCIATES, $3^{\text {a }}$ ed., 2001.

HU, J.; GAO, J.; PRINCIPE, J. C. Analysis of biomedical signals by the Lempel-Ziv complexity: The effect of finite data size. IEEE Transactions on Biomedical Engineering, v. 53, n. 12, p. 2606$2609,2006$.

HUDETZ, A. G. et al. Propofol anesthesia reduces Lempel-Ziv complexity of spontaneous brain activity in rats. Neuroscience Letters, v. 628, p. 132-135, 2016.

IBAÑEZ-MOLINA, A. J. et al. P383 Multiscale lempel-ZIV complexity in schizophrenia at rest and while performing a naming task. Clinical Neurophysiology, v. 128, n. 9, p. e302-e302, 2017.

KANG, X. F. et al. Stochastic detection of enantiomers. Journal of the American Chemical Society, v. 128, n. 33, p. 10684-10685, 2006.

LEMPEL, A.; ZIV, J. On the complexity of finite sequences. EEE TRANSACTIONS INFORMATION THEORY, v. 22, p. 75-81, 1976.

MONTAL, M.; MUELLER, P. Formation of bimolecular membranes from lipid monolayers and a study of their electrical properties. Proceedings of the National Academy of Sciences of the United States of America, v. 69, n. 12, p. 3561-3566, 1972.

MOVILEANU, L. et al. Interactions of peptides with a protein pore. Biophysical journal, v. 89, n. 2, p. 1030-45, 2005

NEVES, G. F. et al. $60 \mathrm{~Hz}$ Electric Field Changes the Membrane Potential During Burst Phase in Pancreatic B-Cells: In Silico Analysis. Acta Biotheoretica, v. 62, n. 2, p. 133-143, 2014.

REINER, J. E. et al. Disease detection and management via single nanopore-based sensors. Chemical Reviews, v. 112, n. 12, p. 64316451, 2012.

RODRIGUES, C. G. et al. Mechanism of $\mathrm{KCl}$ enhancement in detection of nonionic polymers by nanopore sensors. Biophysical journal, v. 95, n. 11, p. 5186-5192, 2008.

RODRIGUES, C. G. et al. Hofmeister effect in confined spaces: Halogen ions and single molecule detection. Biophysical Journal, v. 100, n. 12, p. 2929-2935, 2011.

ROBERTSON, J. W. F. et al. Single-molecule mass spectrometry in solution using a solitary nanopore. Proceedings of the National Academy of Sciences of the United States of America, v. 104, n. 20, p. 8207-11, 2007.

RODRIGUES, C. G. et al. Mechanism of $\mathrm{KCl}$ enhancement in detection of nonionic polymers by nanopore sensors. Biophysical journal, v. 95, n. 11, p. 5186-5192, 2008. 